\title{
Monitoring hydraulic fractures: state estimation using an extended Kalman filter
}

\author{
Fernando Alves Rochinha ${ }^{1}$ and Anthony Peirce P $^{2,3}$ \\ ${ }^{1}$ Mechanical Engineering Department, Federal University of Rio de Janeiro, Rio de Janeiro, \\ Brazil \\ 2 Department of Mathematics, University of British Columbia, British Columbia, Canada \\ E-mail: peirce@math.ubc.ca
}

Received 15 May 2009, in final form 26 November 2009

Published 12 January 2010

Online at stacks.iop.org/IP/26/025009

\begin{abstract}
There is considerable interest in using remote elastostatic deformations to identify the evolving geometry of underground fractures that are forced to propagate by the injection of high pressure viscous fluids. These so-called hydraulic fractures are used to increase the permeability in oil and gas reservoirs as well as to pre-fracture ore-bodies for enhanced mineral extraction. The undesirable intrusion of these hydraulic fractures into environmentally sensitive areas or into regions in mines which might pose safety hazards has stimulated the search for techniques to enable the evolving hydraulic fracture geometries to be monitored. Previous approaches to this problem have involved the inversion of the elastostatic data at isolated time steps in the time series provided by tiltmeter measurements of the displacement gradient field at selected points in the elastic medium. At each time step, parameters in simple static models of the fracture (e.g. a single displacement discontinuity) are identified. The approach adopted in this paper is not to regard the sequence of sampled elastostatic data as independent, but rather to treat the data as linked by the coupled elastic-lubrication equations that govern the propagation of the evolving hydraulic fracture. We combine the Extended Kalman Filter (EKF) with features of a recently developed implicit numerical scheme to solve the coupled free boundary problem in order to form a novel algorithm to identify the evolving fracture geometry. Numerical experiments demonstrate that, despite excluding significant physical processes in the forward numerical model, the EKF-numerical algorithm is able to compensate for the un-modeled dynamics by using the information fed back from tiltmeter data. Indeed the proposed algorithm is able to provide reasonably faithful estimates of the fracture geometry, which are shown to converge to the actual hydraulic fracture geometry as the number of tiltmeters is increased. Since the location of tiltmeters can affect the resolution of the method, the algorithm can also
\end{abstract}

3 Author to whom any correspondence should be addressed. 
be used to design the deployment of tiltmeters to optimize the resolution in regions of particular interest.

\section{Introduction}

Hydraulic fractures (HF) are discontinuities induced to propagate in brittle materials by the injection of a viscous fluid. HF are used to increase the fracture networks in mine ore-bodies to enhance mineral extraction $[3,4]$ and are deliberately created in oil and gas reservoirs to enhance the recovery of hydrocarbons $[1,5]$.

The propagation of HF into undesirable locations can have severe safety consequences in the mining industry and can cause considerable loss of hydrocarbons and environmental damage in the oil industry. Thus, in order to improve the efficacy of HF in industrial applications, it is desirable to improve fracture placement by using models to design treatments and by monitoring HF propagation by measuring the deformations they induce. To this end, there has been considerable research effort devoted to understanding the multi-scale behavior of propagating HF. This has led to a number of mathematical models of varying complexity along with asymptotic $[2,6,8-12]$ and numerical $[13,14,15,27]$ solutions. With regard to the feedback and monitoring of HF there is a paucity of information. The quantities that are readily available in a typical fracture treatment include the volume of fluid pumped and the wellbore pressure. Tiltmeters located in the wellbore itself, in neighboring off-set boreholes, or on the earth's surface are also used to monitor the strain gradient field that is induced by the propagating HF [28]. More recently, a combination of tiltmeter measurements with microseismic images have been used [31].

Inversion of the tiltmeter time series has thus-far involved two strategies. First, the fracture evolution is regarded as a sequence of equilibrium states in which the tilt measurements at each time step are used to solve the shape identification problem by inversion of the crack elasticity operator. Since the elliptic elasticity operator smooths the information about the fracture, it can be demonstrated [30] that the typically small number of remote tilt measurements can at best provide reasonably accurate estimates of the first two moments of the fracture opening, while the higher order moments are subject to significant errors. Shape identification from these noisy, higher order moments is an ill-posed inverse problem. In the second approach, the time series has been used to significantly enrich the data in the elastic inversion process [29] using a Bayesian technique to select the best model from a variety of simple models of the fracture.

However, an inversion of elastostatic data that treat each step of the time series as independent of the the others ignores an important component of the problem. Indeed, the fracture configurations at two consecutive time steps are related by the coupled system of integro-PDE that describe the propagation of the HF. The fact that the coupled dynamic model provides a means of relating the very limited individual tilt measurement data substantially improves the prospects for accurate inversion. For this class of problem, the inversion algorithm is now able to exploit all the causally admissible tilt measurements. This substantially mitigates the severe lack of data suffered by the individual elasto-static inverse problems.

In this paper we use an Extended Kalman Filter (EKF) in conjunction with a forward numerical model to provide an algorithm to identify the fracture boundary and width. Since the EKF is designed for the extraction of parameters or state variables from time series, this aspect of the paper is not new. The novelty of the results presented here derives from the application of the EKF to this particular class of highly nonlinear free boundary problems. Typically such evolution models are susceptible to uncertainties in the initial conditions, the model 


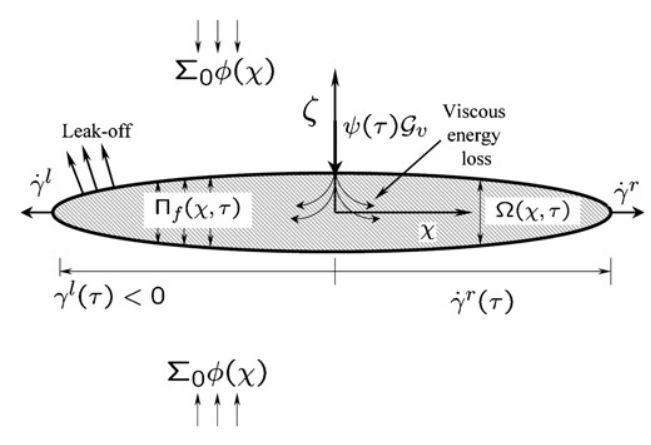

Figure 1. The geometry of the one-dimensional fluid-driven fracture which is assumed to be in a state of plane strain within an elastic medium.

parameters (e.g. the elastic moduli or the in situ stress field) and to the so-called unmodeled dynamics (e.g. physical processes that have been excluded from the modeling process such as fluid leak-off). We demonstrate that the EKF is able to assimilate the low order moment information intrinsic to the tilt measurement time series and feed this information back to the numerical model so that the algorithm is able to estimate the fracture geometry and opening with reasonable precision. We also demonstrate how the tilt placement affects the resolution of the identification algorithm.

In section 2 we describe the forward hydraulic fracture model comprising a system of integro-partial differential equations along with a free-boundary problem and outline a numerical algorithm to locate the free boundary; in section 3 we present the details of the proposed Extended Kalman Filter Numerical (EKFN) algorithm; in section 4 we provide the results of three of numerical experiments chosen to demonstrate the efficacy of the proposed method; in section 5 we provide some concluding remarks.

\section{Hydraulic fracture model}

\subsection{Governing equations and boundary conditions}

In order to test the proposed filtering algorithm we choose a relatively simple model comprising a hydraulic fracture propagating in a state of plane strain [1,9]. This so-called KGD model is able to incorporate a number of important propagation modes, discontinuous in situ stress fields, and Carter leak-off while maintaining a relatively modest computational cost. Since all the numerical experiments presented in this paper involve a dimensionless form of the governing equations we choose, for the sake of brevity, to state the governing equations in a dimensionless form. Detailed descriptions of the dimensional model equations can be found in the literature [10] while we use the same scaling procedure as that used in [27] to define the dimensionless quantities in terms of the characteristic length $\ell_{*}$, time $t_{*}$, pressure $p_{*}$, and width $w_{*}$ that are active in the problem.

We introduce a coordinate system centered on the point source representing the wellbore (see figure 1). The fracture is assumed to occupy the interval $\left(\gamma^{l}, \gamma^{r}\right)$. The dimensionless quantities $\mathcal{G}_{j}$ that appear in the equations below are defined as follows:

$\mathcal{G}_{e}=\frac{E^{\prime} w_{*}}{p_{*} \ell *}, \quad \mathcal{G}_{m}=\frac{w_{*}^{2} p_{*} t_{*}}{\mu^{\prime} \ell_{*}^{2}}, \quad \mathcal{G}_{v}=\frac{Q_{0} t_{*}}{w_{*} \ell_{*}}, \quad \mathcal{G}_{c}=\frac{C^{\prime} t_{*}^{1 / 2}}{w_{*}}, \quad \mathcal{G}_{k}=\frac{K^{\prime} \ell_{*}^{1 / 2}}{E^{\prime} w_{*}}$. 
Here $E^{\prime}=\frac{E}{1-\nu^{2}}$, where $E$ and $\nu$ are the rock Young's modulus and Poisson's ratio; $\mu^{\prime}=12 \mu$, where $\mu$ is the dynamic fluid viscosity; $Q_{0}$ is the volumetric injection rate per unit length in the out-of-plane direction; $C^{\prime}=2 C_{L}$ where $C_{L}$ is Carter's leak-off coefficient; and $K^{\prime}=4\left(\frac{2}{\pi}\right)^{\frac{1}{2}} K_{I C}$ is the modified stress intensity factor.

2.1.1. The elasticity equation. The elastic integral equation [16] relates the net pressure $\Pi(\chi, \tau)$, defined to be the difference between the fluid pressure $\Pi_{f}(\chi, \tau)$ and the opposing ambient geological confining stress $\Sigma_{0} \phi(\chi)$, to the crack opening $\Omega(\chi, \tau)$ according to

$$
\Pi(\chi, \tau)=\Pi_{f}(\chi, \tau)-\Sigma_{0} \phi(\chi)=\frac{\mathcal{G}_{e}}{4 \pi} \int_{\gamma^{l}}^{\gamma^{r}} \frac{\Omega\left(\chi^{\prime}, \tau\right)}{\left(\chi-\chi^{\prime}\right)^{2}} \mathrm{~d} \chi^{\prime} .
$$

For computational efficiency we have chosen this $1 \mathrm{D}$ integral equation formulation rather than solving the 2D Navier equilibrium equations, which would involve volume discretization and costly re-meshing for each growth increment of the evolving HF.

2.1.2. The fluid flow equation. The continuity equation and Poiseuille's law can be combined to yield the following lubrication equation:

$$
\frac{\partial \Omega}{\partial \tau}=\frac{1}{\mathcal{G}_{m}} \frac{\partial}{\partial \chi}\left(\Omega^{3} \frac{\partial \Pi_{f}}{\partial \chi}\right)+\frac{\mathcal{G}_{c} H\left(\tau-\tau_{0}(\chi)\right)}{\sqrt{\tau-\tau_{0}(\chi)}}+\psi(\tau) \mathcal{G}_{v} \delta(\chi), \quad \gamma^{l}(\tau)<\chi<\gamma^{r}(\tau) .
$$

The term involving $\mathcal{G}_{c}$ represents the leak-off of fluid into the surrounding rock since the time $\tau_{0}(\chi)$ at which the fracture tip passed the point $\chi$.

2.1.3. Boundary and propagation conditions. The boundary conditions are that the fracture aperture and fluid-flux should both vanish at the tips:

$$
\Omega\left(\gamma^{l, r}, \tau\right)=0, \quad \lim _{\chi \rightarrow \gamma^{l, r}} \Omega^{3} \frac{\partial \Pi_{f}}{\partial \chi}=0 .
$$

To locate the fracture free boundary we impose an additional propagation condition which requires that the stress intensity factor be in limit equilibrium with the dimensionless fracture toughness $\mathcal{G}_{k}$, which can be expressed by the following asymptotic relation (see [7]):

$$
\lim _{\chi \rightarrow \gamma} \Omega=\mathcal{G}_{k} \sqrt{\gamma-\chi}
$$

Leak-off-storage scaling. If we impose the constraints $\mathcal{G}_{c}=\mathcal{G}_{m}=\mathcal{G}_{e}=\mathcal{G}_{v}=1$, then we obtain four conditions to identify the characteristic quantities $\ell *, t_{*}, p_{*}$ and $w_{*}$. The first of these constraints, equating the dimensionless leak-off coefficient to the dimensionless viscosity, identifies the time $t_{*}$ at which the transition from storage to leak-off dominated regimes occurs. The dimensionless toughness $\mathcal{G}_{k}$ becomes a free parameter in this scaling.

Viscosity-toughness scaling. On the other hand, if we impose the constraints $\mathcal{G}_{k}=\mathcal{G}_{m}=\mathcal{G}_{e}=$ $\mathcal{G}_{v}=1$, then we obtain another set of characteristic quantities in which the $t_{*}$ represents the transition time from viscosity- to toughness-dominated regimes. The dimensionless leak-off coefficient $\mathcal{G}_{c}$ becomes a free parameter in this scaling.

2.1.4. Modes of propagation. The tip asymptote (5) applies if the elastic material ahead of the crack is bonded in which case $\mathcal{G}_{k}>0$. However, if the crack were propagating along the interface between two de-bonded elastic half-spaces so that $\mathcal{G}_{k}=0$, the crack tip asymptote would be different from the square root behavior given in (5). In this case, the appropriate asymptotic behavior can be established by considering a moving coordinate system $\hat{\chi}=\gamma(\tau)-\chi$ and defining new field variables $\Omega(\chi, \tau)=\hat{\Omega}(\gamma(\tau)-\chi)$ and $\Pi(\chi, \tau)=\hat{\Pi}(\gamma(\tau)-\chi)$. There is no loss of generality in assuming that $\gamma(\tau)=\gamma^{r}(\tau)$. 
Ignoring the source and leak-off terms, the fluid-flow equations reduce to the following form:

$$
\dot{\gamma} \frac{\mathrm{d} \hat{\Omega}}{\mathrm{d} \hat{\chi}}=\frac{\mathrm{d}}{\mathrm{d} \hat{\chi}}\left(\hat{\Omega}^{3} \frac{\mathrm{d} \hat{\Pi}}{\mathrm{d} \hat{\chi}}\right) .
$$

Upon integration the lubrication equation reduces to the following form:

$$
\dot{\gamma}=\hat{\Omega}^{2} \frac{\mathrm{d} \hat{\Pi}}{\mathrm{d} \hat{\chi}} .
$$

Now assuming that the width behaves as a power law $\hat{\Omega} \sim A \hat{\chi}^{\alpha}$ as $\hat{\chi} \rightarrow 0$, it can be shown [6] that

$$
\hat{\Pi} \sim \frac{1}{4} A \alpha \cot (\pi \alpha) \hat{x}^{\alpha-1}
$$

Substituting these two asymptotic relations into (6), we obtain

$$
\frac{4 \dot{\gamma}}{\alpha(\alpha-1) \cot (\pi \alpha)}=A \hat{x}^{3 \alpha-2} \text {. }
$$

In order that the right side of this equation should match the constant left side, we require that $\alpha=2 / 3$ and, solving for A, we obtain the leading asymptotic behavior for the width

$$
\hat{\Omega} \sim \beta \dot{\gamma}^{1 / 3} \hat{\chi}^{2 / 3} \quad \text { where } \quad \beta=2^{1 / 3} 3^{5 / 6},
$$

which was first established in [8]. We observe that the primary mechanism for the dissipation of energy for the de-bonded case is that due to driving the viscous fluid through the crack itself. In this paper we assume that the fracture is propagating in the viscosity-dominated regime for which (7) is the relevant asymptote for locating the free boundary at the computational length scale.

\subsection{The discretized forward model}

In this subsection, we describe the procedure used to discretize the governing equations as well as that required to determine the location of the fracture front iteratively. This coupled numerical algorithm is a $1 \mathrm{D}$ version of the implicit level set algorithm [27] that was developed to locate the free boundary of a fracture by exploiting the tip behavior, which has been determined by asymptotic analysis similar to that used to obtain (7) (see [9-12]). This implicit algorithm forms the basis for the forward model used in the inversion experiments. For the purposes of presenting this algorithm we will assume the leak-off-storage scaling so that all the dimensionless parameters $\mathcal{G}_{j}$ except $\mathcal{G}_{k}$ are unity.

The region into which the evolving fracture is expected to propagate is discretized into $N$ uniform elements of length $\Delta \chi=2 a$. The field variables $\Omega(\chi, \tau)$ and $\Pi(\chi, \tau)$ are represented by their values at the centers of the elements. If a fracture tip does not coincide with the edge of an element, then a partially filled tip element is defined in which the width at the center of the element represents the average volume of fluid in the tip. Thus it is possible to represent an evolving HF on a fixed Eulerian grid. Those elements containing the fracture tips form the so-called tip region while those interior elements completely filled with fluid and not containing the tip form the so-called channel region. This logical decomposition of the problem is useful to identify the regions within the evolving fracture in which the appropriate tip asymptotic behavior is imposed as well as those computational mesh points that are used to locate the free boundary. 
2.2.1. The discrete elasticity equation. We assume that $\Omega(\chi, \tau)$ is approximated by a representation in terms of piecewise constant basis functions, i.e. $\Omega(\chi, \tau)=$ $\sum_{m} \Omega_{m}(\tau) H_{m}(\chi)$, where $H_{m}(\chi)$ is the characteristic function for the $m$ th element:

$$
H_{m}(\chi)=\left\{\begin{array}{lll}
1 & \text { if } & \chi \in\left(\chi_{m}-a, \chi_{m}+a\right) \\
0 & \text { if } & \chi \notin\left(\chi_{m}-a, \chi_{m}+a\right)
\end{array}\right.
$$

We then substitute this approximation into the elasticity equation (2), integrate the hypersingular kernel over each element and collocate the equation at element centers. The integral equation is thereby reduced to the following system of linear equations relating the pressures $\Pi_{m}$ and fracture widths $\Omega_{n}$ :

$$
\Pi_{m}(\tau)=\sum_{n} C_{m-n} \Omega_{n}(\tau)
$$

where $C_{m}=\frac{1}{\pi \Delta \chi} \frac{1}{4 m^{2}-1}$.

2.2.2. The discrete fluid-flow equation. To discretize the lubrication equation we integrate (3) over the spacetime interval $\left[\chi_{m}-a, \chi_{m}+a\right] \times[\tau-\Delta \tau, \tau]$. We now approximate the remaining time integrals by the right-hand rule, which, for example for the case of the flux integral, yields the approximation

$\int_{\tau-\Delta \tau}^{\tau}\left[\Omega^{3}\left(\chi, \tau^{\prime}\right) \frac{\partial \Pi_{f}\left(\chi, \tau^{\prime}\right)}{\partial \chi}\right]_{\chi_{k}-a}^{\chi_{k}+a} \mathrm{~d} \tau^{\prime} \simeq \Delta \tau\left[\Omega^{3}(\chi, \tau) \frac{\partial \Pi_{f}(\chi, \tau)}{\partial \chi}\right]_{\chi_{k}-a}^{\chi_{k}+a}$.

We now approximate the spatial integrals of $\Omega$ by the midpoint rule to obtain the following discrete from of the fluid-flow equation:

$$
\Omega_{m}(\tau)=\Omega_{m}(\tau-\Delta \tau)+\Delta \tau\left[A(\Omega(\tau)) \Pi_{f}(\tau)\right]_{m}+\frac{\mathcal{L}_{m}}{\Delta \chi}+\frac{\delta_{m 0}}{\Delta \chi} \Delta \tau \psi(\tau) .
$$

Here $A(\Omega(\tau)) \Pi(\tau)$ is the central difference operator defined by

$$
[A(\Omega(\tau)) \Pi(\tau)]_{k}=\frac{1}{\Delta \chi}\left(\Omega_{k+\frac{1}{2}}^{3}(\tau) \frac{\left(\Pi_{k+1}(\tau)-\Pi_{k}(\tau)\right)}{\Delta \chi}-\Omega_{k-\frac{1}{2}}^{3}(\tau) \frac{\left(\Pi_{k}(\tau)-\Pi_{k-1}(\tau)\right)}{\Delta \chi}\right),
$$

where the half-node values $\Omega_{k \pm \frac{1}{2}}$ are defined to be

$$
\Omega_{k \pm \frac{1}{2}}=\left(\frac{\Omega_{k \pm 1}+\Omega_{k}}{2}\right) \text {. }
$$

The leak-off term $\mathcal{L}_{m}$ is defined as

$$
\mathcal{L}_{m}=2 \int_{\chi_{m}-a}^{\chi_{m}+a}\left(\sqrt{\tau-\tau_{0}(\chi)}-\sqrt{\tau-\Delta \tau-\tau_{0}(\chi)}\right) \mathrm{d} \chi .
$$

These approximations result in a Backward Euler time-stepping scheme, whose $L$-stability is necessary due to the stiffness of the coupled equations.

2.2.3. Locating the free boundary using tip asymptotics. Assuming that the fracture is propagating in the viscosity-dominated regime, we now describe the procedure that can be used to locate the free boundary using the asymptotic expansion (7). The starting point is to invert (7) to obtain

$$
\hat{\chi} \sim\left(\frac{\hat{\Omega}}{\beta \dot{\gamma}^{1 / 3}}\right)^{\frac{3}{2}}
$$

We observe that this asymptotic relation involves the normal velocity $\dot{\gamma}$ of the front. Determining the normal velocity by taking a divided difference approximation to the singular 
pressure gradient is undesirable as it involves an indeterminate limit. As an alternative, the local front velocity can be expressed in terms of two successive front locations $\hat{\chi}_{0}$ and $\hat{\chi}$ :

$$
\dot{\gamma}=\frac{\hat{\chi}-\hat{\chi}_{0}}{\Delta \tau}
$$

We substitute (11) into (10) to eliminate the velocity $\dot{\gamma}$ and rearrange terms to obtain the following cubic equation for the location $\hat{\chi}$ of the front:

$$
\hat{\chi}^{3}-\hat{\chi}_{0} \hat{\chi}^{2}-\Delta \tau\left(\frac{\hat{\Omega}}{\beta}\right)^{3}=0 .
$$

2.2.4. Implicit scheme. The coupled equations (8), (9) and (12) are a nonlinear system of equations, which we solve by Newton iteration, to yield an implicit scheme to locate the free boundary points $\gamma^{l / r}(\tau)$ as well as to determine the field variables $\Omega(\chi, \tau)$ and $\Pi(\chi, \tau)$.

\section{Hydraulic fracture monitoring as a nonstationary inverse problem}

Data assimilation [17, 18] has been used in different fields either for calibrating models through parameter identification or for estimating states, or both. These formulations blend information provided by physical models with observed, often incomplete, noisy data obtained either from experiments or from field monitoring.

Traditional data assimilation methods rely upon the Kalman Filter and extensions of this method. The main ideas, which are based on the use of Bayesian Inference, are described in detail in $[19,20]$. The next section features a brief presentation of the basic equations and concepts. Indeed, Extended Kalman Filters (EKF) have been extensively employed for inverse problems related to a wide range of applications (see for example [21-24]) and, particularly relevant for the present work [25], in which the EKF was applied to crack detection.

\subsection{A brief review of the EKF method}

An EKF estimates the state evolution of a physical system, within a probabilistic framework, through the systematic use of an evolution model and observed data, which is assumed to be corrupted by noise and can also be incomplete. Therefore, the goal, in the present context, is to obtain the state (here represented by discrete versions of $\Omega(\chi, \tau), \Pi(\chi, \tau)$ and the unknown fracture domain $\left[\gamma^{l}, \gamma^{r}\right]$ ) at each instant of the fracture evolution with the help of deformation measurements and the discrete evolution model introduced in the previous section.

The filter is built upon a discrete state-space representation of the system expressed in the following compact form:

$$
\begin{aligned}
& X_{k+1}=F_{k+1}\left(X_{k}, W_{k+1}\right) \\
& Y_{k+1}=G_{k+1}\left(X_{k+1}, V_{k+1}\right) .
\end{aligned}
$$

Here $X_{k}=\left[\gamma^{l}, \gamma^{r}, \Omega(\chi, \tau), \Pi(\chi, \tau)\right]$ is a vector containing the states at instant $\tau_{k}, Y_{k}$ is the vector of observations provided by the tiltmeters, and $F$ and $G$ are the evolution and the observation models, respectively. The corresponding operators are nonlinear. Moreover $W_{k+1}$ and $V_{k+1}$ are stochastic processes introduced in order to account for possible uncertainties. The former are associated with modeling errors that might be caused by the spacetime discretization of the continuous model or by un-modeled dynamics that might result from a failure to account for some significant physical process in the formulation of the idealized model, while the latter operator is associated with the noise typically produced by measurements. With the intent 
of producing a real-time monitoring scheme for the present application, the model $F$ is constructed using the numerical scheme described in section 2 to solve the coupled system of integro-partial differential equations (2)-(5).

The EKF comprises two stages, namely prediction and update. The first stage corresponds to solving (13) on the time interval $\left[\tau_{k}, \tau_{k+1}\right]$. The explicit form of equation (13) is adopted here to follow the general notation often encountered in the literature, but it is worth mentioning that the fracture domain is only defined and computed implicitly through the model introduced previously. The second stage consists of inverting the observed data in order to obtain an estimation of the state vector at $\tau_{k+1}$.

Evolving these two stages in time corresponds to a sequential identification algorithm which is summarized below. The results of this algorithm are the estimated values of the state vector at each instant and the corresponding measures of uncertainty associated with the estimation provided by the state covariance matrix $\Gamma$. In order to identify the values of the variables within the different stages of the algorithm, we adopt a notation with a double subscript - reminiscent of that used to denote conditional probabilities. Thus, $X_{\tau_{k} \mid \tau_{j}}$ represents the estimation of the state at $\tau_{k}$ taking into consideration data observed at time $\tau_{j}(j \leqslant k)$.

- Prediction: given $X_{k \mid k}$ and $\Gamma_{k \mid k}$ compute

$$
\begin{aligned}
& X_{k+1 \mid k}=F_{k+1}\left(X_{k \mid k}\right) \\
& \Gamma_{k+1 \mid k}=\mathcal{D} F_{k+1 \mid k} \Gamma_{k \mid k} \mathcal{D} F_{k+1 \mid k}^{T}+\Gamma_{w_{k+1}} .
\end{aligned}
$$

- Measurement update (data inversion):

$$
\begin{aligned}
& K_{k+1}=\Gamma_{k+1 \mid k} J_{k+1}^{T}\left(J_{k+1} \Gamma_{k+1 \mid k} J_{k+1}^{T}+\Gamma_{v_{k+1}}\right)^{-1} \\
& X_{k+1 \mid k+1}=X_{k+1 \mid k}+K_{k+1}\left(Y_{k+1}-G\left(X_{k+1 \mid k}\right)\right) \\
& \Gamma_{k+1 \mid k+1}=\left(I_{d}-K_{k+1} J_{k+1}\right) \Gamma_{k+1 \mid k},
\end{aligned}
$$

where $\mathcal{D} F_{k+1}$ is the Jacobian of $F$ computed at $X_{k \mid k}$, frequently referred to as the transition matrix, and $\Gamma_{w_{k+1}}$ is the covariance matrix associated with the noise process $W$. The matrix $K$ is the so-called Kalman Gain and $J_{k+1}$ is the Jacobian of the observation operator $G . \Gamma_{v_{k+1}}$ is the covariance matrix corresponding to the noise in the measurements. The superscripts $T$ and -1 denote, respectively, transpose and inverse matrices.

The sequential identification algorithm described above requires knowledge of the initial state and its covariance. It also requires models for describing uncertainties to be attributed to the observation and evolution stages as well. These details will be addressed later.

\subsection{An EKF applied to 1D-hydraulic fracture}

We now combine the EKF algorithm described above with the numerical model outlined in section 2 to produce an algorithm to identify the state evolution of a hydraulic fracture, which is assumed to be propagating in a state of plane strain. In what follows we will refer to this algorithm as the EKFN algorithm in order to emphasize that the filter is built upon a numerical model. The hydraulic fracture is constrained to grow along a line which is taken, without loss of generality, to be the horizontal axis. Since the source-point of the fracture, corresponding to the wellbore, is typically assumed to be known, the objective of the filter is to retrieve the fracture domain defined by the two extremities $\gamma^{r}$ and $\gamma^{l}$ over the time interval $\left[\tau_{0}, \mathcal{T}\right]$. Moreover, the fracture opening, described by the function $\Omega(\chi, \tau)$ (with $\chi \in\left(\gamma^{l}, \gamma^{r}\right)$ ), also needs to be identified. The pressure distribution $\Pi(\chi, \tau)$, which is important in the design and evaluation of the stimulation, can be obtained from $\Omega(\chi, \tau), \gamma^{l}$ and $\gamma^{r}$ by using the 
elasticity operator given in (2) or its discrete form (8). The identification process assumes that the following parameters are known: the injected flow rate $Q_{0}$, the elastic parameters of the surrounding medium $E$ and $\nu$, and the viscosity of the fracturing fluid $\mu$.

3.2.1. Evolution model. The evolution model to be employed here consists of the discretized model equations described in section 2. The EKFN requires the solution of this nonlinear problem at each time step. The state associated with the hydraulic fracture corresponds to the vector field $X=\left[\gamma^{l}, \gamma^{r}, \Omega\right]^{T}$, where $\Omega$ is the vector of fracture apertures sampled at the mesh points of the discrete model. Using the procedure described in section 2.2 to locate the free boundary by inverting the asymptoptic relation (7), the locations of the free boundary points $\gamma_{k+1 \mid k+1}^{l}$ and $\gamma_{k+1 \mid k+1}^{r}$ can be determined from the width $\Omega$. It is therefore possible to define a reduced state vector $X_{k+1 \mid k+1}$ comprising only the unknown fracture widths $\Omega_{k+1 \mid k+1}$. In this case the tangent map $\mathcal{D} F$ corresponds to the Jacobian of the nonlinear system (8), (9).

3.2.2. Observation model. The observation model uses the strain field generated by the fracture in the surrounding elastic medium. More specifically, the observation model to be used with the EKFN combines the elasticity equation and measurements provided by tiltmeters [28]. These sensors, which are deployed either on the surface or along offset observation boreholes, measure the inclination induced by the induced strain field. The measured tilt angles associated with the horizontal $(\chi)$ and vertical $(\zeta)$ directions defined relative to the reference plane, are given by

$$
\omega_{i}=\omega\left(\chi_{i}, \zeta_{i}\right)=\frac{\partial u_{\zeta}}{\partial \chi}-\frac{\partial u_{\chi}}{\partial \zeta} \quad i=1, N \quad \text { sites }
$$

where $\left(\chi_{i}, \zeta_{i}\right)$ are the coordinates of the observation sites and $u=\left(u_{\chi}, u_{\zeta}\right)$ is the displacement field. Thus, the observation model is cast in the form of the following integral equation:

$$
\omega\left(\chi_{i}, \zeta_{i}\right)=-\frac{2}{\pi} \mathcal{G}_{s} \int_{\gamma^{l}}^{\gamma^{r}} \frac{\chi^{\prime} \zeta_{i} \Omega\left(\chi^{\prime}\right)}{\left[\left(\chi_{i}-\chi^{\prime}\right)^{2}+\left(\zeta_{i}\right)^{2}\right]^{2}} \mathrm{~d} \chi^{\prime}
$$

with $\mathcal{G}_{s}=\frac{w_{*}}{\ell_{*}}$.

At this point, it is worth noting that the above equation involves a nonlinear relation between the crack boundary points $\gamma^{l}$ and $\gamma^{r}$ and the measured inclinations, which corresponds to a nonlinear elastic inverse problem. From that perspective, the EKFN could be interpreted as a regularization scheme that provides prior information embedded in the states furnished by the evolution model at each time instant.

The data inversion, which constitutes a second stage filter, requires the computation of $J$, the Jacobian of the integral operator defined in (20) evaluated at the predicted state $X_{k+1 \mid k}$, which was computed in the first stage. The first step in obtaining $J$ involves the directional derivative given by

$D_{\delta X_{k}} \omega\left(X_{k}\right)=\int_{\gamma^{l}}^{\gamma^{r}} \tilde{G}\left(\chi, \chi^{\prime}\right) \delta \Omega_{k} \mathrm{~d} \chi^{\prime}+\tilde{G}\left(\chi, \gamma_{k}^{r}\right) \Omega_{k}\left(\gamma_{k}^{r}\right)-\tilde{G}\left(\chi, \gamma_{k}^{l}\right) \Omega_{k}\left(\gamma_{k}^{l}\right)$

with $\tilde{G}$ representing the kernel of the operator introduced in the integrand of relation (20).

Due to the vanishing width boundary conditions (4), the last two terms of (21) are zero, which implies that the inclinations are not sensitive to first order perturbations associated with changes in the fracture domain. Therefore, updating $\gamma^{l, r}$ does not follow the procedure employed by standard EKF implementations. Indeed, we choose to use the reduced state vector $X_{k+1 \mid k+1}=\Omega_{k+1 \mid k+1}$, in the EKFN formulation and to locate the free boundary points by inverting the asymptotic relation (7).

The main challenges related to the monitoring of hydraulic fractures by directly inverting data from tiltmeters using models of the form (20) have been addressed in detail in [28]. These 
authors analyze the limitations of such an inverse formulation, particularly when tiltmeters are placed far away from the hydraulic fracture itself - a situation which typically occurs whenever surface tiltmeters are used. Indeed only global parameters such as the fracture orientation and volume can be obtained with any precision. Similar conclusions are drawn in [29, 30] where only elastostatic data are used for the inversion. At this point, it is important to emphasize that the approach introduced here goes beyond inverting the sequence of quasi-static elastic snapshots because the data are coupled to an evolution model. This makes it possible to solve for the crack boundaries even though these terms are not explicitly present in the linearized equation (21).

3.2.3. Summary of the EKFN algorithm. We briefly summarize the main steps of the proposed filter algorithm that starts from an initial state which is assumed to be known and combines the readings from tiltmeters with the predictions provided by the implicit algorithm described in section 2.2 .

\section{EKFN algorithm}

- Initialize the state : $k=1, \quad X_{1}$.

- Advance time step : $k=k+1, \quad \tau \leftarrow \tau+\Delta \tau$. Solve forward model (8), (9) and (12) for $\gamma^{l, r}$ and $\Omega$.

Given $\gamma^{l, r}$ and $\Omega$, compute $\mathcal{D} F$.

Update the covariance matrix $\Gamma$ using (16).

Compute the Jacobian $J$ using (21).

Compute the Kalman Gain matrix $K$ using (17).

Update the state variables using (18).

Update the covariance matrix $\Gamma$ using (19).

- End time step loop.

\section{Results}

In this section we present results for three distinct examples which have been chosen to illustrate the efficacy as well as the shortcomings of the proposed monitoring technique. All the examples presented use noisy synthetic tiltmeter data produced by the numerical scheme presented in section 2. The typical scenario is depicted schematically in figure 2, where tiltmeters are placed either on the surface or along offset monitoring wells.

In order not to commit the so-called inverse crime [20], the simulated data have been computed subject to the following constraints: the data were obtained using a more dense mesh than the one used for the state estimation; for some of the situations, the time step was also assumed to be different from that used to generate the synthetic data; zero-mean white noise was added to the tiltmeter outputs with standard deviation varying from $1 \%$ to $5 \%$ of the maximum value measured. This last condition implies that the matrix $V_{k}$ introduced in (14) is diagonal, having each non-zero entry defined as stated before.

In initial verification tests, which are not presented here, the EKFN algorithm was able to faithfully reproduce both the fracture width and the tip locations for a HF propagating in a uniform in situ confining stress field without leak-off. A significantly coarser mesh was used for the EKFN algorithm than that used to generate the synthetic data. These results are to be expected, since the forward model itself, even without feedback from the tilt measurements via the EKF, should provide some approximation to the HF used to generate the synthetic data.

In order to challenge the EKFN algorithm, we present results for three numerical experiments in which the model used to generate the synthetic data contains a significant 


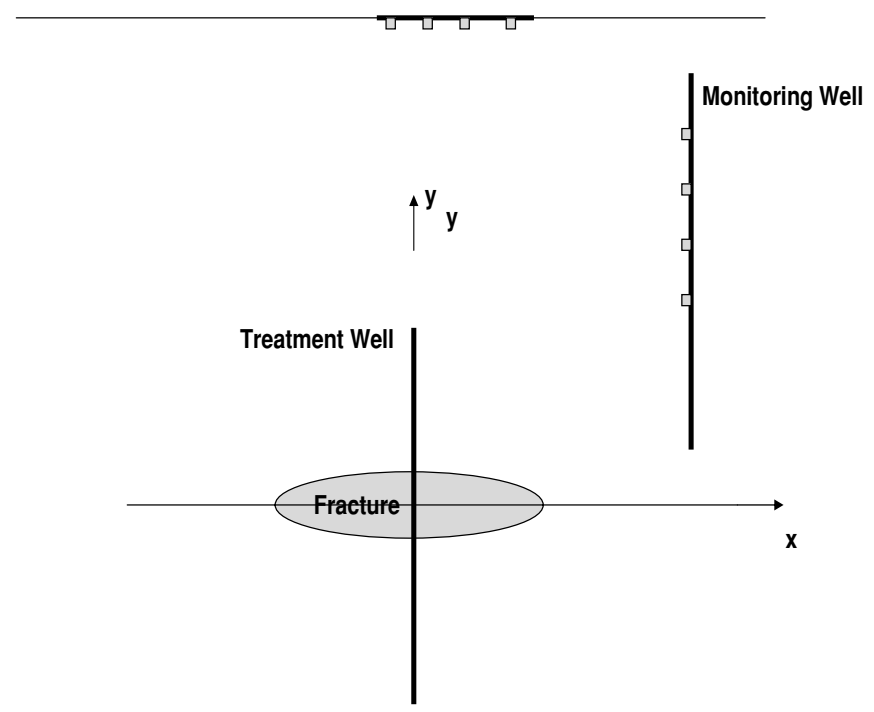

Figure 2. Schematic view of a typical monitoring configuration.

parametric variation or a dominant physical process which is omitted from the forward numerical model used in the EKFN. In the first experiment, the confining stress field $\Sigma_{0} \phi(\chi)$ for the synthetic data is assumed to decrease linearly with increasing $\chi$, whereas the forward model used for the EKFN assumes a uniform confinement field. In the second experiment, the synthetic confinement field has two jump discontinuities while the EKFN confinement field is assumed to be uniform. In the third experiment, the synthetic data are generated by a model in which significant leak-off is present whereas the EKFN forward model assumes that the rock is impermeable. The first two examples may be classified as having parametric uncertainty, while the third example involves un-modeled dynamics, as a dominant physical process has been ignored in the forward model. In each case the covariance of the model and initial conditions were assumed to be diagonal matrices. The covariance associated with the observation model mainly depends on the measurement systems and is typically estimated from experiments or field observations. On the other hand, the covariance of the evolution model, which incorporates uncertainties in the modeling, is not easily evaluated. Successful attempts that rely either on experiments [32] or on analytical tools [33] reveal the complexity of this task. Here we adopt a frequently employed pragmatic strategy, which assumes that the covariances are proportional to the reference state values. As the forward model is in a non-dimensional form, the states are typically $O(1)$ and we assume that the covariance is 0.01 for the simulations shown below. Indeed, for both the observation and evolution models, several numerical experiments were carried out in order to assess the dependence of the results on the choice of the covariances. In all cases similar results were obtained, which indicates the robustness of the proposed methodology with respect to the choice of the covariance values adopted here.

In all the experiments it was found that the location of the tiltmeters can have a significant impact on the efficacy of the method. As expected, the performance of the EKFN depends on the relative location of the advancing fracture tips and the tiltmeter stations, on the number of measurement sites and the extent to which they generate data which is independent. This situation is not static either, since, as the fracture evolves, the tilt array can move into and out 


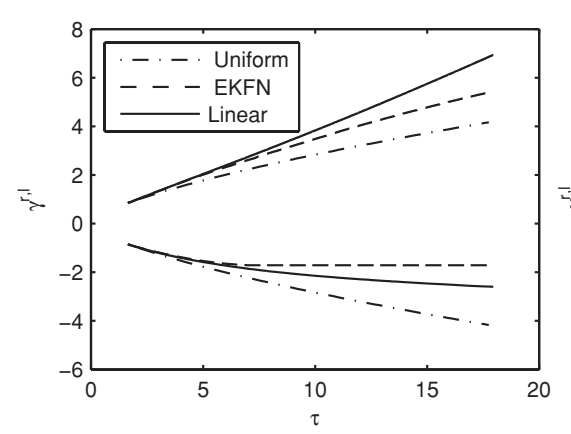

(a) 2-tilt EKFN estimates

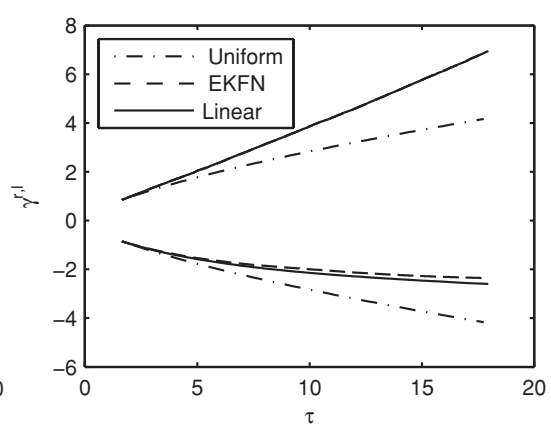

(b) 5-tilt EKFN estimates

Figure 3. Fracture tip positions for linearly varying and uniform in situ stress fields. The corresponding EKFN-uniform estimates of the tip positions are also shown.

of an advantageous position for measurement. This poses a challenge when deciding which results to present-do we use only the worst case results or the best results? In an attempt to provide a somewhat dispassionate assessment of the algorithm we have chosen to present results for arrays of two, three or five tiltmeters to illustrate the limitations and the possibilities of the technique. In practice, the placement of the tiltmeters to provide resolution in a region of interest could, in itself, become the subject of simulation and optimization in the design of the deployment of the monitoring apparatus.

\subsection{Fracture evolution in a linear in situ stress field}

The synthetic data were generated assuming that $\Sigma_{0} \phi(\chi)=\alpha_{0}-\alpha_{1} \chi$, where $\alpha_{0}=1$ and $\alpha_{1}=0.01$. The synthetic model started at an initial fracture with a radius of $-\gamma^{l}=\gamma^{r}=0.85$ corresponding to a dimensionless time $\tau=1.63$. The solution given by Carbonell [26] was used to initialize the numerical algorithm, which was assumed to propagate in a viscositydominated regime. A mesh size of $\Delta \chi=0.1$ and a time step of $\Delta \tau=0.0102$ were used. The forward model used by the EKFN assumes a uniform in situ stress field $\Sigma_{0} \phi(\chi)=1$, uses a mesh size $\Delta \chi=0.2$ and the same time step, and is assumed to start from the same initial solution as the synthetic solution but sampled on the coarser grid.

Such a linear variation in the in situ stress field is typical in relatively homogeneous regions underground in which the increase in stress with depth is due to the overburden rock. The growth of a hydraulic fracture in such a stress field is asymmetric as it follows the trajectory of least resistance.

In figure 3(a) we plot the fracture lengths $\gamma^{l}<0$ and $\gamma^{r}>0$ for the synthetic model with a linear in situ stress field, the forward model with a uniform stress field, and the EKFNuniform estimates using feedback from two tiltmeters located at $\left(\chi_{1}, \zeta_{1}\right)=(0 ., 0.9238)$ and $\left(\chi_{2}, \zeta_{2}\right)=(2 ., 0.9238)$. Even with this small number of tiltmeter stations, the EKFN algorithm is able to detect and compensate for the asymmetry in the fracture growth. We also note that the left tip is estimated a little more accurately than the right tip, which is probably due to the distance between the crack tip and the sensor array.

In figure 3(b) we plot the same data as in figure 3(a) except that the EKFN model now uses an array of five sensors constructed by adding three tiltmeters located at $\left(\chi_{3}, \zeta_{3}\right)=$ $(4 ., 0.9238),\left(\chi_{4}, \zeta_{4}\right)=(6 ., 0.9238)$ and $\left(\chi_{5}, \zeta_{5}\right)=(8 ., 0.9238)$ to the 2 -sensor array. In this case the additional tiltmeters yield estimates for the right tip location that is indistinguishable 


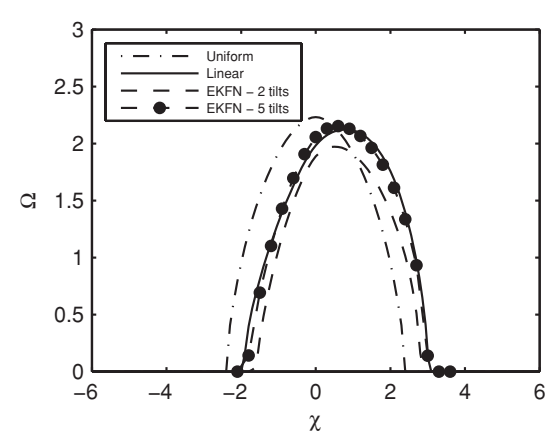

(a) Small time $\tau=600$

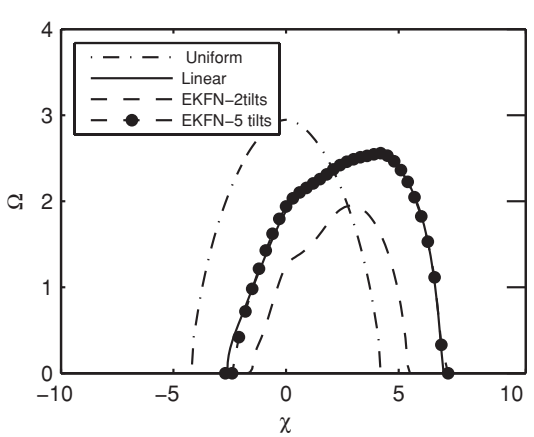

(b) Large time $\tau=1600$

Figure 4. Fracture openings $\Omega$ for linearly varying and uniform in situ stress fields. The corresponding EKFN-uniform estimates of the fracture opening using two and five tiltmeters are shown.

from the synthetic values, while there is a small improvement in the estimate of the left tip position.

In figures 4(a) and (b) we compare synthetic, uniform and EKFN-uniform estimates of the fracture openings $\Omega$ at early $(\tau=600)$ and advanced $(\tau=1600)$ sample times in the simulation. The EKFN solution using feedback from the five-tiltmeter array provides a good estimate of the synthetic fracture opening throughout the simulation. The EKFN solution using two tilts is reasonably accurate initially, but exhibits significant spurious leak-off as the simulation progresses.

\subsection{Fracture evolution in a discontinuous stress field}

For this example synthetic data were generated assuming that the in situ stress field has the following piecewise continuous behavior:

$$
\Sigma_{0} \phi(\chi)= \begin{cases}0.6 & \text { for } \chi<-3 \\ 0.5 & \text { for }-3 .<\chi<3 \\ 0.3 & \text { for } 3 .<\chi\end{cases}
$$

The wellbore is located at $\chi=0$ so the fracture propagates symmetrically until the stress discontinuities are encountered at $|\chi|=3$. Since the confining stress is larger for $\chi<-3$. than it is for $\chi>3$, the fracture will propagate preferentially across the right-most stress jump rather than to the left. In fact, since the confining stress in the region $\chi>3$. is smaller than that in the interval $-3 .<\chi<3$., the fracture will even tend to herniate into the region $\chi>3$. Such piecewise constant stress fields are common underground due to the sedimentary deposition and genesis of the layered rock strata.

The synthetic model started with an initial fracture having a radius of $-\gamma^{l}=\gamma^{r}=2.75$ corresponding to a dimensionless time $\tau=9.4924$. A mesh size of $\Delta \chi=0.5$ and a time step $\Delta \tau=0.0949$ were used. The forward model used by the EKFN assumes a uniform in situ stress field $\Sigma_{0} \phi(\chi)=0.5$, a mesh size $\Delta \chi=0.5$, the same time step and is assumed to start from the same initial solution as the synthetic solution.

In figure 5(a) we plot the fracture lengths $\gamma^{l}<0$ and $\gamma^{r}>0$ for the synthetic model with the discontinuous in situ stress field defined above, the forward model with a uniform stress field and the EKFN-uniform estimates using feedback from two tiltmeters located at $\left(\chi_{1}, \zeta_{1}\right)=(0 ., 0.9238)$ and $\left(\chi_{2}, \zeta_{2}\right)=(2 ., 0.9238)$. The EKFN algorithm provides a 


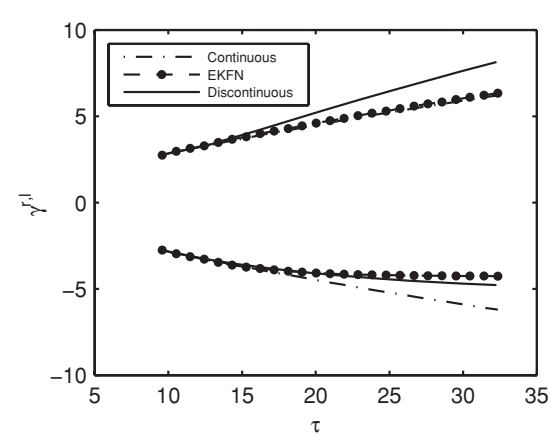

(a) 2-tilts

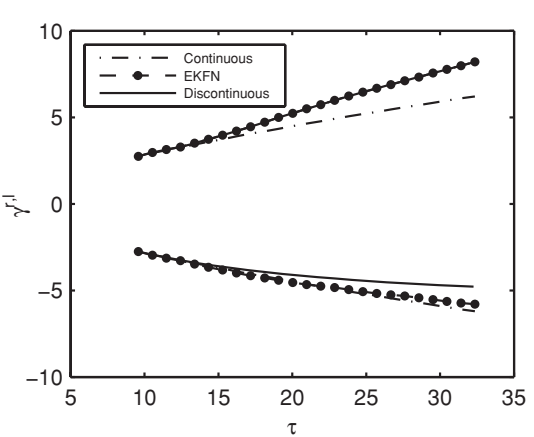

(b) 5-tilts

Figure 5. Fracture tip positions for discontinuous and uniform in situ stress fields. The corresponding EKFN-uniform estimates using two and five tiltmeters are shown.
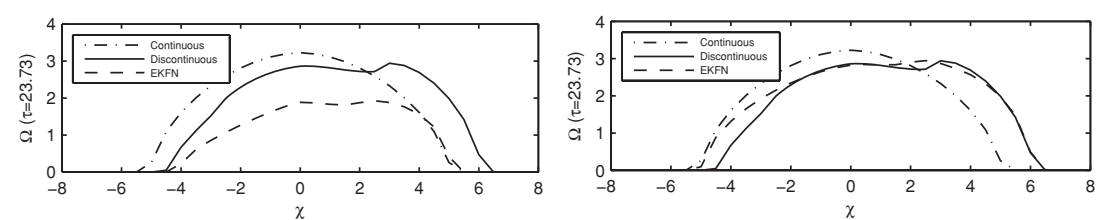

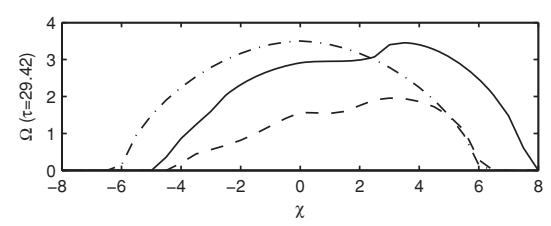

(a) 2-tilts

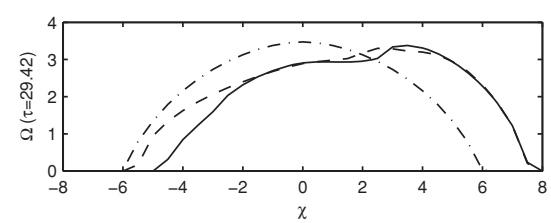

(b) 5-tilts

Figure 6. Fracture openings for discontinuous and uniform in situ stress fields at two sample times in the simulation. The corresponding EKFN-uniform estimates of the fracture openings using the two- and five-tiltmeter arrays are shown.

reasonable location of the left tip while it produces a poor location of the right tip, which seems to track the right tip position associated with a uniform in situ stress field.

By augmenting the sensor array with three additional tiltmeters located at $\left(\chi_{3}, \zeta_{3}\right)=$ (4., 0.9328), $\left(\chi_{4}, \zeta_{4}\right)=(6 ., 0.9328)$ and $\left(\chi_{5}, \zeta_{5}\right)=(8 ., 0.9328)$, the location of the right tip is significantly improved, see figure $5(\mathrm{~b})$, while the location of the left tip position actually deteriorates. This somewhat surprising result is probably due to the spurious leak-off in the case of the 2-sensor array (see figure 6(a)), which results in an estimation of the less rapidly advancing left tip which only seems to be more accurate.

In figures 6(a) and (b) we compare the fracture openings $\Omega$ of the synthetic, uniform and EKFN-uniform estimates using two- and five-tiltmeter arrays, respectively. In each figure the fracture openings $\Omega$ at an early $\tau=23.73$ and later $\tau=29.42$ time are plotted. The EKFNuniform solution using feedback from the five-tiltmeter array is clearly an improvement on the estimate given by the two-tiltmeter array.

The above results demonstrate that the proposed method performs well even in the presence of significant amounts of noise. Indeed, similar results were obtained when the initial configurations provided to the filter were perturbed. Besides, a relatively small number of sensors were employed and were located sufficiently far from the fracture so that the configuration could be interpreted as monitoring the fracture evolution from the surface. 


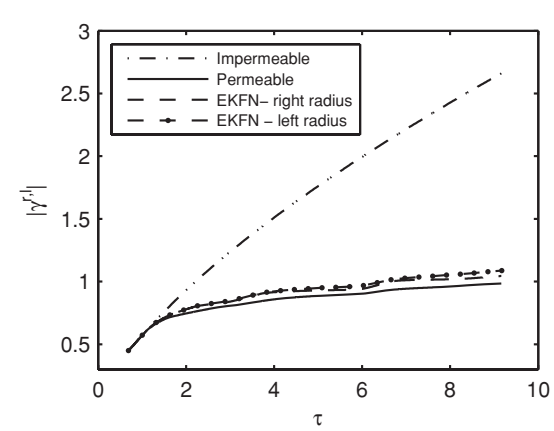

(a) Fracture tip positions

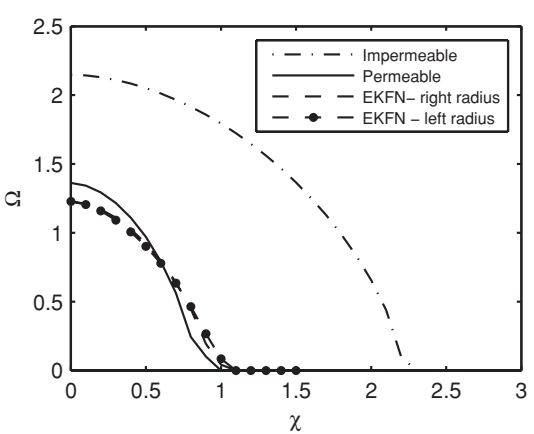

(b) Opening

Figure 7. Fracture tip positions (left) and fracture openings (right) for permeable and impermeable media. The EKFN-impermeable estimates with three tilt measurements are shown.

\subsection{Hydraulic fracture propagation with leak-off}

In the previous two examples the HF were assumed to be propagating in impermeable media. In this example, the synthetic data are produced by the $1 \mathrm{D}$ numerical model in a permeable medium in which we assume that the dimensionless leak-off coefficient $\mathcal{G}_{c}=1$. The synthetic model started with an initial fracture having a radius of $-\gamma^{l}=\gamma^{r}=0.45$ corresponding to a dimensionless time $\tau=0.688$. A mesh size of $\Delta \chi=0.1$ and a time step of $\Delta \tau=0.0079$ were used. The forward model used by the EKFN assumes no leak-off, a mesh size $\Delta \chi=0.2$, the same time step and is assumed to start from the same initial solution as the synthetic solution but sampled on the coarser grid. Thus a significant component of the physical situation has been omitted from the forward model used by the EKFN.

In figure 7(a) we plot the fracture lengths $-\gamma^{l}$ and $\gamma^{r}$ for the synthetic model with leak-off, the forward model without leak-off and the EKFN-impermeable estimates using feedback from three tiltmeters located at $\left(\chi_{1}, \zeta_{1}\right)=(0 ., 0.9328),\left(\chi_{2}, \zeta_{2}\right)=(2 ., 0.9328)$ and $\left(\chi_{3}, \zeta_{3}\right)=(4 ., 0.9328)$. The left and right fracture lengths for the synthetic permeable and the impermeable model have identical left and right fracture lengths due to the symmetry of the problem. The EKFN-impermeable estimates of the left an right tip positions are not identical because of the asymmetric location of the tiltmeter array relative to the wellbore. We observe that the EKFN estimates of the tip positions are remarkably close to the actual locations used to generate the synthetic data.

In figure 7(b) we compare the fracture openings $\Omega$ of the synthetic permeable, impermeable and EKFN-impermeable estimates using the three-tiltmeter array. In each case, the fracture openings $\Omega$ are sampled at the time $\tau=6.95$. The EKFN-impermeable fracture opening using feedback from the three-tiltmeter array shows excellent agreement with that of the simulated data in which the medium was assumed to be permeable. Thus, in spite of the dominant unmodeled dynamics, which is the cause of the huge discrepancy between the permeable and impermeable $\Omega$ values, the feedback from the tiltmeter data via the EKFN is able to compensate and to provide excellent estimates of the fracture opening and tip positions.

\section{Conclusions}

The real-time monitoring of propagating HF is important for industrial applications in which it is desirable to avoid the penetration of the HF into environmentally sensitive regions, for 
example. Hitherto, the inversion of tiltmeter data has focussed on the inversion of elastostatic data sampled at snapshots taken during the well stimulation process. These measurements were used to estimate the parameters in very simplified models of the fracture plane-such as an isolated displacement discontinuity or the identification of the moments of the fracture width. Because of the limited number of tiltmeters that can be deployed and the constraints on their location, there is little data for the purposes of inversion. This lack of information and the fact that the elasticity operator rapidly smooths the strain field with distance from the fracture, conspire to make the inverse problem ill-posed.

In this paper we have explored the possibility of connecting these isolated elastostatic measurements through a coupled elasto-lubrication forward model for the evolution of the HF itself. This approach means that all the causally admissible measurements can be deployed to determine the desired information about the evolving fracture geometry. We have explored the use of the EKF combined with a discrete coupled model based on the implicit level set algorithm in order to identify the fracture geometry. Data from the tilt measurement time series is fed back to the forward model to provide corrections for parameter uncertainty or unmodeled dynamics.

Since we are using a forward model in the inversion process, there is an expectation that the forward model itself might produce viable estimates of the fracture geometry. In the examples presented we have chosen to deliberately challenge the EKFN algorithm by ignoring significant parameter variations or physical processes that have a dominant effect on the fracture geometry. In spite of these hurdles, the EKFN was able to identify the fracture geometry with remarkable fidelity given the relatively few tiltmeter measurements that were used.

As is to be expected, we found that the the number and location of the tiltmeter arrays can have a significant impact on the resolution of the EKFN. Although this is a limitation of the algorithm, it does open the possibility of using the algorithm itself to determine the optimal tiltmeter location before deployment in the field in order to achieve, for example, the best resolution in a particular region of interest.

\section{Acknowledgments}

AP gratefully acknowledges the support of the NSERC Discovery Grants Program. FR acknowledges the support provided by $\mathrm{CNPq}$ during his sabbatical leave, when the present work was initiated.

\section{References}

[1] Adachi J, Siebrits E, Peirce A and Desroches J 2007 Computer simulation of hydraulic fractures Int. J. Rock Mech. Min. Syst. 44 739-57

[2] Lister J R 1990 Buoyancy-driven fluid fracture: the effects of material toughness and of low-viscosity precursors J. Fluid Mech. 210 263-80

[3] Jeffrey R G and Mills K W 2000 Hydraulic fracturing applied to inducing longwall coal mine goaf falls Pacific Rocks 2000 (Rotterdam: Balkema) pp 423-30

[4] van As A and Jeffrey R G 2000 Caving induced by hydraulic fracturing at North Parkes mines Pacific Rocks 2000 (Rotterdam: Balkema) pp 353-60

[5] Al Matar M, Al Mutawa M, Aslam M, Dashti M, Sharma J, Byung OL, Solares R, Nemec T, Sware J and Tealdi L 2008 The right treatment for the right reservoir Oilfield Rev. 20

[6] Spence D A and Sharp P 1985 Self-similar solutions for elastohydrodynamic cavity flow Proc. R. Soc. Lond. Ser. A 400 289-313

[7] Rice J R 1968 Mathematical analysis in the mechanics of fracture Fracture: An Advanced Treatise ed H Liebowitz (New York: Academic) chapter 3 pp 191-311 
[8] Desroches J, Detournay E, Lenoach B, Papanastasiou P, Pearson J R A, Thiercelin M and Cheng A 1994 The crack tip region in hydraulic fracturing Proc. R. Soc. Lond. Ser. A 447 39-48

[9] Detournay E 2004 Propagation regimes of fluid-driven fractures in impermeable rocks Int. J. Geomech. 4 1-11

[10] Adachi J I and Detournay E 2008 Plane-strain propagation of a fluid-driven fracture in a permeable medium Eng. Fract. Mech. 75 4666-94

[11] Garagash D I and Detournay E 2005 Plane-strain propagation of a fluid-driven fracture: small toughness solution ASME J. Appl. Mech. 72 916-28

[12] Mitchell S L, Kuske R and Peirce A P 2007 An asymptotic framework for the analysis of hydraulic fractures: the impermeable case ASME J. Appl. Mech. 74 365-72

[13] Clifton R J and Abou-Sayed A S 1979 On the computation of the three-dimensional geometry of hydraulic fractures Proc. of the SPE Symp. on Low Permeability Gas Reservoirs (Denver, Richardson TX, SPE7943, Society of Petroleum Engineers) pp 307-13

[14] Peirce A P 2006 Localized Jacobian ILU preconditioners for hydraulic fractures Int. J. Numer. Methods Eng. 65 1935-46

[15] Peirce A P and Siebrits E 2005 A dual mesh multigrid preconditioner for the efficient solution of hydraulically driven fracture problems Int. J. Numer. Methods Eng. 63 1797-823

[16] Hills D A, Kelly P A, Dai D N and Korsunsky A M 1996 Solution of crack problems The Distributed Dislocation Technique, Solid Mechanics and Its Applications vol 44 (Dordrecht: Kluwer)

[17] Evensen G 2007 Data Assimilation: The Ensemble Kalman Filter (Berlin: Springer)

[18] Kalnay E 2003 Atmospheric Modelling, Data Assimilation and Predictability (Cambridge: Cambridge University Press)

[19] Anderson B D O and Moore J B 1979 Optimal Filtering (New York: Dover)

[20] Kaipio J and Somersalo E 2005 Statistical and Computational Inverse Problems (Applied Mathematical Sciences vol 160) (Berlin: Springer)

[21] Mandel J, Bennethum L S, Beezley J D, Coen J L, Douglas C C, Kim M and Vodacek A 2008 A wildland fire model with data assimilation Math. Comput. Simul. 79 584-606

[22] Seppanen A, Vauhkonen M, Vauhknone P J, Voutilainen A and Kaipio J P 2007 State estimation in process tomography-three-dimensional impedance imaging of moving fluids Int. J. Numer. Methods Eng. 73 2141-57

[23] Ijaz U Z, Khambampati A K, Lee J S, Kim S and Kim K Y 2008 Nonstationary phase boundary estimation in electrical impedance tomography using unscented Kalman filter J. Comput. Phys. 227 7089-112

[24] Ojima Y and Kawahara M 2009 Estimation of river current using reduced Kalman filter finite element method Comput. Methods Appl. Mech. Eng. 198 904-11

[25] Maier G, Bocciarelli M, Bolzon G and Fedele R 2006 Inverse analyses in fracture mechanics Int. J. Fract. $13847-73$

[26] Carbonell R, Desroches J and Detournay E 1999 A comparison between a semi-analytical and a numerical solution of a two-dimensional hydraulic fracture Int. J. Solids Struct. 36 4869-88

[27] Peirce A and Detournay E 2008 An implicit level set method for modeling hydraulically driven fractures Comput. Methods Appl. Mech. 197 2858-85

[28] Lecampion B, Jeffrey R and Detournay E 2005 Resolving the geometry of hydraulic fractures from tilt measurements Pure Appl. Geophys. 162 2433-52

[29] Lecampion B and Gunning J 2007 Model selection in fracture mapping from elastostatic data Int. J. Solid Struct. 44 1391-408

[30] Lecampiaon B and Peirce A 2007 Multipole moment decomposition for imaging hydraulic fractures from remote elastostatic data Inverse Problem 23 1641-58

[31] Warpinski N R, Griffin L G, Davis E J and Grant T 2006 Improving hydraulic frac diagnostics by joint inversion of downhole microseismic and tiltmeter data Paper SPE 102690 presented at 2006 SPE Annual Technical Conference and Exhibition (San Antonio, TX)

[32] Heikkinen L M, Vilhunen T, West R M and Vauhkonen M 2002 Simultaneous reconstruction of electrode contact impedances and internal electrode properties: II. Laboratory experiments Meas. Sci. Technol. 13 1855-61

[33] Huttunen J M J and Kaipio J P 2007 Approximation errors in nonstationary inverse problems Inverse Problems Imaging 177-93 